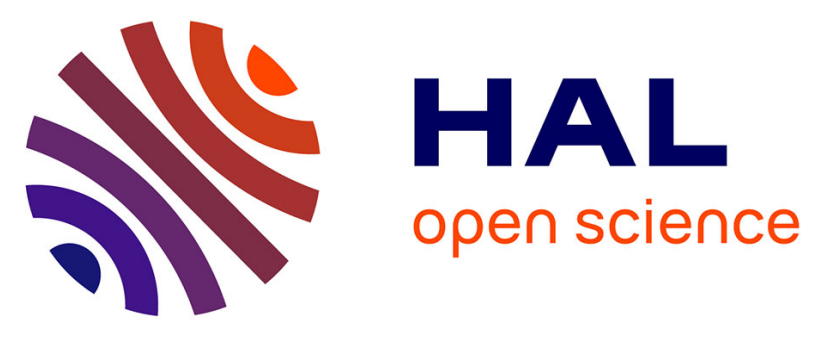

\title{
ON THE COMPLEMENTARITY OF PULSAR TIMING AND SPACE LASER INTERFEROMETRY FOR THE INDIVIDUAL DETECTION OF SUPERMASSIVE BLACK HOLE BINARIES
}

Alessandro D. A. M. Spallicci, Patrick François

\section{To cite this version:}

Alessandro D. A. M. Spallicci, Patrick François. ON THE COMPLEMENTARITY OF PULSAR TIMING AND SPACE LASER INTERFEROMETRY FOR THE INDIVIDUAL DETECTION OF SUPERMASSIVE BLACK HOLE BINARIES. The Astrophysical journal letters, 2013, 764 (2), pp.187. 10.1088/0004-637X/764/2/187 . insu-01258145

\section{HAL Id: insu-01258145 \\ https://hal-insu.archives-ouvertes.fr/insu-01258145}

Submitted on 18 Jan 2016

HAL is a multi-disciplinary open access archive for the deposit and dissemination of scientific research documents, whether they are published or not. The documents may come from teaching and research institutions in France or abroad, or from public or private research centers.
L'archive ouverte pluridisciplinaire HAL, est destinée au dépôt et à la diffusion de documents scientifiques de niveau recherche, publiés ou non, émanant des établissements d'enseignement et de recherche français ou étrangers, des laboratoires publics ou privés.

\section{(이)(\$)}

Distributed under a Creative Commons Attribution - NonCommercial - NoDerivatives 4.0 


\title{
ON THE COMPLEMENTARITY OF PULSAR TIMING AND SPACE LASER INTERFEROMETRY FOR THE INDIVIDUAL DETECTION OF SUPERMASSIVE BLACK HOLE BINARIES
}

\author{
Alessandro D. A. M. Spallicci \\ Université d'Orléans, Observatoire des Sciences de l'Univers en région Centre, UMS 3116 LPC2E-CNRS UMR 7328, \\ 3A Av. Recherche Scientifique, F-45071 Orléans, France; spallicci@cnrs-orleans.fr \\ Received 2012 October 7; accepted 2013 January 13; published 2013 February 6
}

\begin{abstract}
Gravitational waves coming from supermassive black hole binaries (SMBHBs) are targeted by both the Pulsar Timing Array (PTA) and Space Laser Interferometry (SLI). The possibility of a single SMBHB being tracked first by PTA, through inspiral, and later by SLI, up to merger and ring-down, has been previously suggested. Although the bounding parameters are drawn by the current PTA or the upcoming Square Kilometer Array (SKA), and by the New Gravitational Observatory (NGO), derived from the Laser Interferometer Space Antenna (LISA), this paper also addresses sequential detection beyond specific project constraints. We consider PTA-SKA, which is sensitive from $10^{-9}$ to $p \times 10^{-7} \mathrm{~Hz}(p=4,8)$, and SLI, which operates from $s \times 10^{-5}$ up to $1 \mathrm{~Hz}(s=1,3)$. An SMBHB in the range of $2 \times 10^{8}-2 \times 10^{9} M_{\odot}$ (the masses are normalized to a $(1+z)$ factor, the redshift lying between $z=0.2$ and $z=1.5$ ) moves from the PTA-SKA to the SLI band over a period ranging from two months to fifty years. By combining three supermassive black hole (SMBH)-host relations with three accretion prescriptions, nine astrophysical scenarios are formed. They are then related to three levels of pulsar timing residuals $(50,5,1 \mathrm{~ns})$, generating 27 cases. For residuals of $1 \mathrm{~ns}$, sequential detection probability will never be better than $4.7 \times 10^{-4} \mathrm{yr}^{-2}$ or $3.3 \times 10^{-6} \mathrm{yr}^{-2}$ (per year to merger and per year of survey), according to the best and worst astrophysical scenarios, respectively; put differently this means one sequential detection every 46 or 550 years for an equivalent maximum time to merger and duration of the survey. The chances of sequential detection are further reduced by increasing values of the $s$ parameter (they vanish for $s=10$ ) and of the SLI noise, and by decreasing values of the remnant spin. The spread in the predictions diminishes when timing precision is improved or the SLI low-frequency cutoff is lowered. So while transit times and the SLI signal-to-noise ratio $(\mathrm{S} / \mathrm{N})$ may be adequate, the likelihood of sequential detection is severely hampered by the current estimates on the number-just a handful—of individual inspirals observable by PTA-SKA, and to a lesser extent by the wide gap between the pulsar timing and space interferometry bands, and by the severe requirements on pulsar timing residuals. Optimization of future operational scenarios for SKA and SLI is briefly dealt with, since a detection of even a single event would be of paramount importance for the understanding of SMBHBs and of the astrophysical processes connected to their formation and evolution.
\end{abstract}

Key words: black hole physics - galaxies: nuclei - gravitational waves - pulsars: general - space vehicles

Online-only material: color figure

\section{INTRODUCTION}

At different paces, Earth-based and space gravitational wave detectors and observatories are already-or will soon beoperating. They aim to cover different parts of the gravitational spectrum, targeting an extremely large variety of astrophysical sources. Nonetheless, large gaps will show between the sensitive frequency bands. One such gap is that between the Pulsar Timing Array (PTA) - in the future the Square Kilometer Array (SKA) - and the Space Laser Interferometry (SLI) bands. SLI may materialize as the New Gravitational Observatory $(N G O)$, derived from the Laser Interferometer Space Antenna (LISA). Though their bands lie apart, PTA-SKA and SLI may monitor the same type of source, namely a supermassive black hole binary (SMBHB) at different evolutionary stages. In this paper, we explore under what circumstances a single SMBHB may be viewed first by PTA-SKA and later by SLI.

The motivation for the study lies in the opportunities offered by the analysis of the same sources with different instruments (radio astronomy and interferometry), and at different relativistic regimes (inspiral, coalescence, merger, and ring-down). If pulsar timing were to provide mass and spin parameters, the latter could be counter-checked by laser interferometry. Likewise, a binary system could be examined, vis-à-vis the presence of matter, gas, and other bodies, at different phases of its evolution.

\subsection{Observation by Pulsar Timing}

In the frequency band between $10^{-9} \mathrm{~Hz}$ and some fraction of $10^{-6} \mathrm{~Hz}$, PTA offers the unique chance to observe gravitational radiation. Beyond the observation of a stochastic background, the challenge of observing a single SMBHB by PTA has recently received growing attention. Simulations concur in predicting a scenario wherein some SMBHBs stick out of the stochastic signal floor of unresolved SMBHBs. The observation of an individual SMBHB provides opportunities for new measurements in general relativity and new perspectives for the scientific community.

The state of the art on timing residual precision ${ }^{1}$ from current PTAs $^{2}$ lies in the $100-50 \mathrm{~ns}$ domain, while improvement by SKA $^{3}$ down to $10-1 \mathrm{~ns}$ is expected (Liu et al. 2011).

\footnotetext{
1 The timing residuals are computed from the phase difference between the observed time of arrival (ToA) and the predicted ToA, based on the current model parameters.

2 PPTA: http://www.atnf.csiro.au/research/pulsar/ppta, EPTA: http://www.epta.eu.org/, and NANOGrav: http://nanograv.org/.

3 SKA: http://www.skatelescope.org/.
} 
Investigations ${ }^{4}$ on individual sources aim to recover physical parameters such as the spins and the masses of, and the distance to, an SMBHB (Sesana \& Vecchio 2010) using the gravitational wave front curvature (Deng \& Finn 2011), or the Pulsar term ${ }^{5}$ (Corbin \& Cornish 2010; Lee et al. 2011; Mingarelli et al. 2012). From these studies it emerges that the distance to a pulsar is important for individual SMBHBs, unlike statistical background observation (Jenet et al. 2004; Sesana et al. 2009; Finn \& Lommen 2010). Finn \& Lommen (2010) and Pitkin (2012) analyzed bursts coming from different sources including individual SMBHBs.

Burt et al. (2011) suggest refraining from searching for new pulsars, unless close to an existing cluster of good pulsars; instead, they recommend the allocation of more observation time to already low-noise pulsars.

Turning to specific observation targets, searches have so far produced negative results: Jenet et al. (2004) found no evidence of the emission of gravitational waves by a supposed SMBHB in 3C 66B; neither did Lommen \& Backer (2001), who were seeking evidence for an SMBHB in Sgr A*.

Finally, Yardley et al. (2010) describe the observations used to produce the sensitivity curves for the Parkes radio telescope and propose a method for detecting significant sinusoids in PTA.

\subsection{Detection by Space Laser Interferometry}

Gravitational wave detection in space was first proposed by means of a small-sized interferometer on board a single satellite (Grassi-Strini et al. 1979), before shaping into a triangular satellite configuration (Bertotti 1984; Faller et al. 1985, 1989). The LISA pathfinder (Antonucci et al. 2012) is deemed an important step toward technological maturity.

$N G O^{6}$ (ESA 2011), which is derived from the previous LISA proposal, ${ }^{7}$ is a space project designed to measure gravitational radiation over a broadband at low frequencies, where the universe is richly populated by strong sources of gravitational waves, including SMBHBs. NGO plans to trace the formation, growth, and merger history of supermassive black holes (SMBHs) during different epochs, measuring spin and masses, with an unprecedented precision, often where the universe is blind with our current electromagnetic techniques (ESA 2011). In fundamental physics, different tests on general relativity, including the no-hair theorem and the dynamics in strong field, and on alternative theories will be feasible with SLI. NGO (AmaroSeoane et al. 2012a, 2012b) implies a shift to higher frequencies of the sensitivity band, as compared to LISA.

\subsection{Sequential Detection}

Pitkin et al. (2008) first proposed sequential detection, but it appeared necessary to improve and update their initial work for several reasons. We begin by ascertaining that the total SMBHB mass is generally larger than the 50 million solar masses considered by Pitkin et al. (2008). New analyses are carried out herein. First, we examine various astrophysical scenarios

\footnotetext{
4 In the following cited studies, two simplifying hypotheses have been adopted: (1) binaries are on circular orbits and (2) the mergers are gravitational wave driven.

5 The "Earth term" is the gravitational wave strain at the Earth at the time when the pulse is received. The "Pulsar term" is the strain at the pulsar at the time when the pulse is emitted. An SMBHB produces two

quasi-monochromatic components in PTA residuals, and likely of different frequencies as the SMBHB evolves.

6 http://sci.esa.int/ngo

7 http://sci.esa.int/lisa
}

combining SMBH-host relations and accretion processes, and dry and wet mergers. Second, we span a large range of residuals (50-1 ns). Third, we estimate the number of events and the probability of sequential detection, building our investigation upon the recent statistical findings of individual detection by PTA-SKA, which were not available at the time of the work by Pitkin et al. (2008). Fourth, we present the signal-to-noise ratio $(\mathrm{S} / \mathrm{N})$ of SLI for the sources concerned.

\subsection{Structure of the Paper}

Section 2 is devoted to the computation of transit times (how long it takes for a binary to switch bands) from the PTA-SKA to the SLI band for a range of SMBHB masses. In the same section, the ringing frequencies are computed for the (stationary and rotating) SMBHB remnants.

Section 3 is the core of the paper, in which we study the impact of astrophysical, observational, and detection constraints separately, one step after the other, steadily building up our analysis. Details of the models are described by Sesana et al. (2009), to which the reader is referred for further information. SMBH-host relation models are then combined with accretion prescription models, and different values of timing residuals are considered. We determine first the maximum number of sequential detections within a given time to merger, assuming that SLI would catch all the sources that were previously observed by PTA-SKA. We then consider the impact of the SLI low cutoff frequency and of the spin of the remnants. Then for $N G O$, we compute the $\mathrm{S} / \mathrm{N}$ at different values of the redshift $z$, and we finally adapt our previous estimates for sequential detection.

Section 4 sums up the conclusions; the Appendix attempts to sketch some of the operational scenarios that may lie ahead.

We refer to the total mass of the binary $M$, normalized to $1+z$, where $z$ is the redshift (Hughes 2002). The mass enters in the orbit evolution equation with the timescale $\mathrm{Gm} / \mathrm{c}^{3}$, the timescale being redshifted. The consequence ${ }^{8}$ is that a binary at $z=1.5$ and of mass $2 \times 10^{8} M_{\odot}$ is equivalent to a binary at $z=0.2$ and of mass $4.17 \times 10^{8} M_{\odot}$. Herein, given the range of $z$, the factor $(1+z)$ introduces an uncertainty of less than 2.25 on the mass determination.

We use the terms of observation and detection when referring to PTA-SKA and SLI, respectively, while sequential detection implies both observation by PTA-SKA and detection by SLI. Finally, the term "event" refers mostly to astrophysical phenomena, or it is used whenever a specific labeling is not intended.

\section{TRANSIT TIME AND RINGING}

Although notable advances in the two-body problem have been achieved (Blanchet et al. 2011), the foundations laid by Peters \& Mathews (1963) and Peters (1964) - the PM model — still suffice for the description of the relativistic binaries for the present analysis. The main assumptions (Pierro \& Pinto 1996; Pierro et al. 2001) of the PM model are as follows: (1) point masses, (2) weak field, (3) slow motion, and (4) adiabatic evolution (negligible change in the orbital parameters over each orbit).

In the PM model, the time it takes a circularized binary, of equal masses $m_{1}=m_{2}=m$ and total mass $m_{1}+m_{2}=M$, to

\footnotetext{
8 Petiteau et al. (2011) discuss the feasibility of breaking the degeneracy with
} electromagnetic counterparts and propose enforcing statistical consistency. 


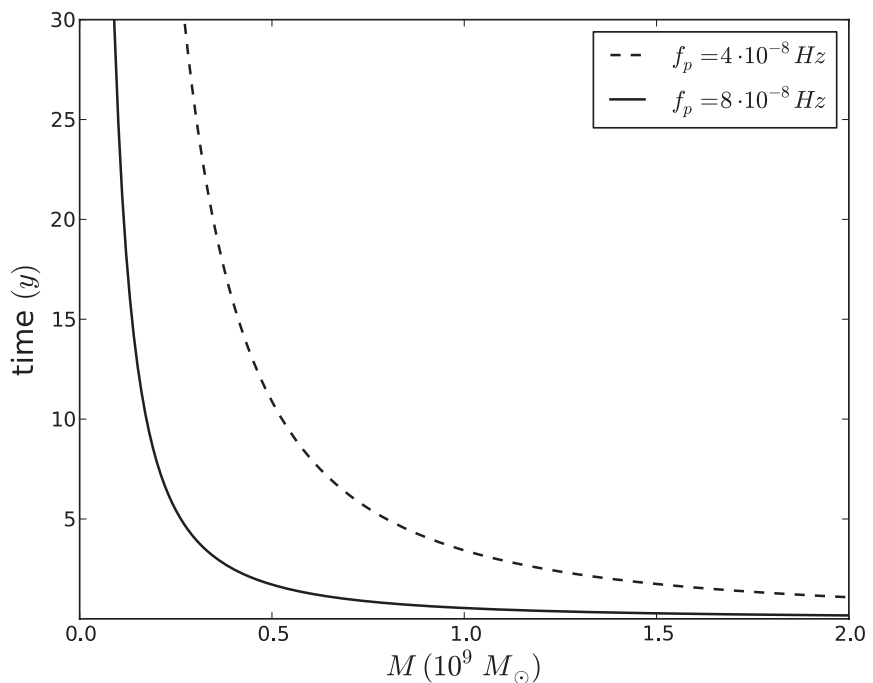

Figure 1. Time of transit (years) as a function of the SMBHB total normalized mass, from the PTA-SKA sensitivity band $\left(f_{p}=4 \times 10^{-7} \mathrm{~Hz}\right.$, dashed line, or $8 \times 10^{-7} \mathrm{~Hz}$, solid line) to the SLI sensitivity band $\left(f_{s}=2 \times 10^{-5} \mathrm{~Hz}\right)$.

evolve between two frequencies is given $b^{9}$

$$
t_{t}=t_{p}-t_{s}=\kappa M^{-5 / 3}\left(f_{p}^{-8 / 3}-f_{s}^{-8 / 3}\right),
$$

where $t_{t}$ is the time of transit from the PTA-SKA band (exited at $t_{p}$ time) to the SLI band (entered at $t_{s}$ time); $f_{p}$ and $f_{s}$ are the PTA-SKA high-frequency and the SLI low-frequency cutoffs, respectively; the numerical coefficient is given by

$$
\kappa=5 \times 2^{-35 / 9} \pi^{-8 / 3}\left(\frac{G}{c^{3}}\right)^{-5 / 3},
$$

with $G$ being the constant of gravitation and $c$ the speed of light.

The $f_{p}$ cutoff is determined by the interval between observations with the radio telescope. A daily allocation would bring the cutoff to $10^{-5} \mathrm{~Hz}$. We have taken a semi-conservative stand by setting two values for $f_{p}$, namely $4 \times 10^{-7} \mathrm{~Hz}$ and $8 \times 10^{-7} \mathrm{~Hz}$. If $f_{s} \gg f_{p}$, the value of $f_{s}$ becomes irrelevant in the computation of the transit time. In this regard, a shift toward higher frequencies of the band of SLI is not consequential. Conversely, the chances for sequential detection are strongly dependent on even a slight shift of $f_{s}$, and further, if the space interferometer has a modest sensitivity at low frequencies, the sequential detection may be easily missed; see Section 3. Figure 1 shows the transit time.

The coalescence frequency, the frequency $f_{c}$ at which the post-Newtonian expansion of the inspiral ceases to be accurate at around $6 M$ (Hughes 2002), and the ring-down frequency $f_{r}$ (Echeverria 1989; Hughes 2002) are given by

$$
\begin{gathered}
f_{c} \simeq 4 \times 10^{-6} \frac{10^{9} M_{\odot}}{M} \mathrm{~Hz}, \\
f_{r} \simeq 3.2 \times 10^{-5} \frac{10^{9} M_{\odot}}{\eta M}\left[1-0.63(1-a)^{3 / 10}\right] \mathrm{Hz} .
\end{gathered}
$$

The ring-down frequencies fall within the band of some of the SLI configurations, especially for a high spin Kerr parameter $a$;

\footnotetext{
9 Equation (1) is obtained by integrating Equation (4) in Forward \& Berman (1967), as Peters \& Mathews did not write an expression for the frequency evolution.
}

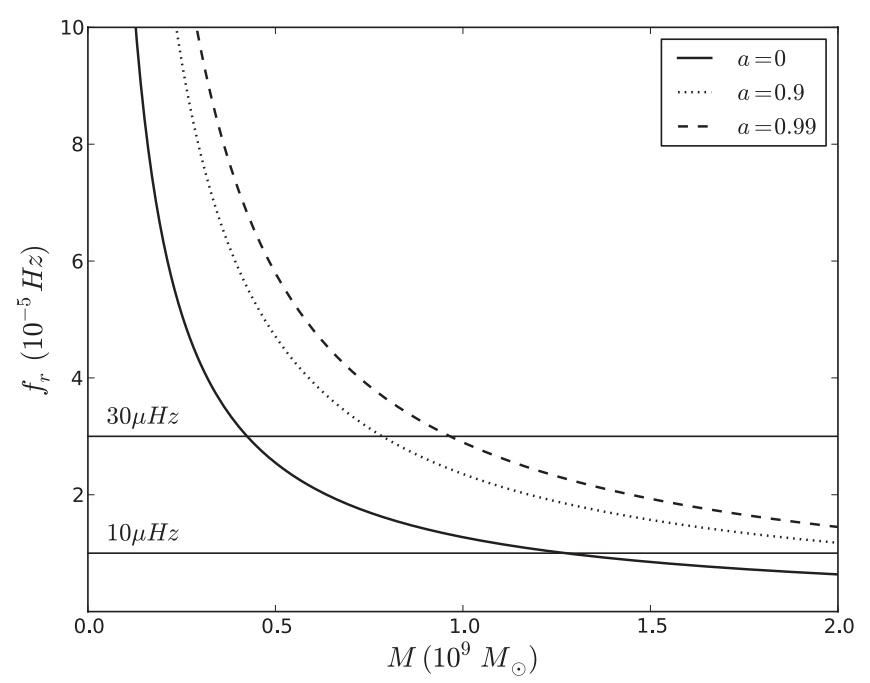

Figure 2. Frequency $(\mathrm{Hz})$ of ringing as a function of the final SMBH total normalized mass, for three dimensionless spin Kerr parameter $a$ values: 0 (solid line), 0.9 (dotted line), and 0.99 (dashed line). The horizontal lines correspond to two different values of $f_{s}$, i.e., 10 or $30 \mu \mathrm{Hz}$.

see Figure 2. The parameter $\eta=0.94$ takes into account the emission of gravitational radiation. ${ }^{10}$

Transit time and ring-down frequencies appear compatible with sequential detection. The shortest transit time is determined primarily by the $f_{p}$ frequency, while the longest transit time is determined by the interval chosen between observation by PTA-SKA and detection by SLI (see the Appendix).

\section{OBSERVATION AND DETECTION RATES AND PROBABILITIES, SIGNAL-TO-NOISE RATIO}

In this section, we combine three SMBH-host relations with three accretion prescriptions. The nine scenarios are further coupled with three different values of timing residuals, producing 27 cases. For each of these cases, we retain the SMBHBs that generate a timing residual above a given threshold $(50,5,1 \mathrm{~ns})$. There are two timescales: $t_{r}$, which fixes the maximum time to merger that we intend to observe, and $\Delta t_{l}$ which refers to the SLI survey duration, i.e., the SLI mission lifetime (see the Appendix).

First, we compute the number of observations of those SMBHBs which are individually detectable by PTA-SKA, and which are up to 10 years away from merger (see Table 1): these values include those SMBHBs which would not enter any currently considered SLI band, as they are too massive. Conversely, if we imagine that the PTA-SKA and SLI bands are contiguous, the values in Table 1 take on the meaning of ideal upper values. Then, we derive a sub-set of the previous ensemble of SMBHBs whose remnants all enter the SLI band, for two different values of the SLI low cutoff frequencies and for different remnant spins (see Tables 2 and 3). An optimal sequential detection rate corresponds to this sub-set, having assumed so far a noiseless interferometer. Finally, for the same sources, we focus on $N G O$ and compute the $\mathrm{S} / \mathrm{N}$ at different values of the redshift $z$ in the range of 0.2-1.5, where the observable SMBHBs are expected to be (see Table 4; Sesana et al. 2009). Lastly, we comment on our previous estimates for sequential detection, in light of the characteristics (noise and cutoff) of the $N G O$ project.

\footnotetext{
10 According to numerical simulations, the mass radiated in gravitational waves is $M_{\mathrm{rad}} / M=1-M_{\mathrm{fin}} / M=5-7 \times 10^{-2}$, with $M=M_{1}+M_{2}$ being the total mass (Rezzolla 2009).
} 
Table 1

PTA-SKA Observation Rates (Ideal Sequential Detection Upper Values)

\begin{tabular}{lccc}
\hline \hline Model & $50 \mathrm{~ns}$ & $5 \mathrm{~ns}$ & $1 \mathrm{~ns}$ \\
\hline $\mathrm{Aa}$ & $3.5 \times 10^{-9}$ & $3.4 \times 10^{-6}$ & $3.3 \times 10^{-4}$ \\
$\mathrm{Ab}$ & $1.4 \times 10^{-8}$ & $1.8 \times 10^{-5}$ & $1.5 \times 10^{-3}$ \\
$\mathrm{Ac}$ & $9.5 \times 10^{-8}$ & $1.2 \times 10^{-4}$ & $5.5 \times 10^{-3}$ \\
$\mathrm{Ba}$ & $8.7 \times 10^{-9}$ & $2.1 \times 10^{-5}$ & $9.1 \times 10^{-4}$ \\
$\mathrm{Bb}$ & $2.3 \times 10^{-7}$ & $1.4 \times 10^{-4}$ & $4.0 \times 10^{-3}$ \\
$\mathrm{Bc}$ & $3.9 \times 10^{-6}$ & $1.3 \times 10^{-3}$ & $1.6 \times 10^{-2}$ \\
$\mathrm{Ca}$ & $8.2 \times 10^{-7}$ & $3.3 \times 10^{-4}$ & $4.3 \times 10^{-3}$ \\
$\mathrm{Cb}$ & $1.5 \times 10^{-5}$ & $1.6 \times 10^{-3}$ & $1.5 \times 10^{-2}$ \\
$\mathrm{Cc}$ & $3.5 \times 10^{-4}$ & $9.7 \times 10^{-3}$ & $4.7 \times 10^{-2}$ \\
\hline
\end{tabular}

Notes. Total number of PTA-SKA observations in 10 years $\left(n_{10,10}\right.$ units) for SMBHBs individually observable and generating a timing residual above a given threshold $(50,5,1 \mathrm{~ns})$, merging in less than 10 years, and not necessarily destined to enter the SLI band, if too massive. Three models of SMBH-host relations and three accretion types have been explored, producing nine crosscombinations, each evaluated for three timing residual options. The resolvable SMBHBs are mostly situated at a redshift $0.2<z<1.5$.

Table 2

Optimal Sequential Detection Rates

\begin{tabular}{lccc}
\hline \hline Model & $\begin{array}{c}\text { All Spins } \\
\text { at } a=0\end{array}$ & $\begin{array}{c}\text { All Spins } \\
\text { at } a=0.9\end{array}$ & $\begin{array}{c}\text { All Spins } \\
\text { at } a=0.99\end{array}$ \\
\hline $\mathrm{Aa}$ & $1.8 \times 10^{-4}$ & $2.9 \times 10^{-4}$ & $3.1 \times 10^{-4}$ \\
$\mathrm{Ab}$ & $3.2 \times 10^{-4}$ & $8.0 \times 10^{-4}$ & $9.7 \times 10^{-4}$ \\
$\mathrm{Ac}$ & $8.7 \times 10^{-4}$ & $2.5 \times 10^{-3}$ & $3.1 \times 10^{-3}$ \\
$\mathrm{Ba}$ & $5.33 \times 10^{-4}$ & $1.18 \times 10^{-3}$ & $1.4 \times 10^{-3}$ \\
$\mathrm{Bb}$ & $8.56 \times 10^{-4}$ & $2.71 \times 10^{-3}$ & $3.56 \times 10^{-3}$ \\
$\mathrm{Bc}$ & $2.42 \times 10^{-3}$ & $9.11 \times 10^{-3}$ & $1.25 \times 10^{-2}$ \\
$\mathrm{Ca}$ & $9.65 \times 10^{-4}$ & $3.15 \times 10^{-3}$ & $4.11 \times 10^{-3}$ \\
$\mathrm{Cb}$ & $1.12 \times 10^{-3}$ & $4.48 \times 10^{-3}$ & $6.64 \times 10^{-3}$ \\
$\mathrm{Cc}$ & $3.48 \times 10^{-3}$ & $1.59 \times 10^{-2}$ & $2.4 \times 10^{-2}$ \\
\hline
\end{tabular}

Notes. For the nine astrophysical scenarios, optimal (noiseless SLI) sequential detection rate, $n_{10,10}$ units, or the total number of events in 10 years, for SMBHBs (1) observed by PTA-SKA at $1 \mathrm{~ns}$ level, (2) entering the SLI bandwidth, the $f_{s}$ cutoff frequency being at $10^{-5} \mathrm{~Hz}$, (3) merging in less than 10 years, and (4) for an SLI survey duration of 10 years. The SMBHBs are mostly situated at a redshift $0.2<z<1.5$. All the remnants have identical spins (either $a=0$, or 0.9 , or 0.99$)$.

Table 3

Optimal Sequential Detection Rates

\begin{tabular}{lccc}
\hline \hline Model & $\begin{array}{c}\text { All Spins } \\
\text { at } a=0\end{array}$ & $\begin{array}{c}\text { All Spins } \\
\text { at } a=0.9\end{array}$ & $\begin{array}{c}\text { All Spins } \\
\text { at } a=0.99\end{array}$ \\
\hline $\mathrm{Aa}$ & $3.8 \times 10^{-6}$ & $7.1 \times 10^{-5}$ & $1.1 \times 10^{-4}$ \\
$\mathrm{Ab}$ & $2.3 \times 10^{-6}$ & $9.4 \times 10^{-5}$ & $1.7 \times 10^{-4}$ \\
$\mathrm{Ac}$ & $6.1 \times 10^{-6}$ & $2.3 \times 10^{-4}$ & $4.5 \times 10^{-4}$ \\
$\mathrm{Ba}$ & $2.8 \times 10^{-6}$ & $8.5 \times 10^{-5}$ & $1.5 \times 10^{-4}$ \\
$\mathrm{Bb}$ & $2.5 \times 10^{-6}$ & $1.6 \times 10^{-4}$ & $2.0 \times 10^{-4}$ \\
$\mathrm{Bc}$ & $5.6 \times 10^{-6}$ & $2.7 \times 10^{-4}$ & $5.5 \times 10^{-4}$ \\
$\mathrm{Ca}$ & $3.4 \times 10^{-6}$ & $1.2 \times 10^{-4}$ & $2.3 \times 10^{-4}$ \\
$\mathrm{Cb}$ & $2.5 \times 10^{-6}$ & $1.6 \times 10^{-4}$ & $2.4 \times 10^{-4}$ \\
$\mathrm{Cc}$ & $6.9 \times 10^{-6}$ & $3.4 \times 10^{-4}$ & $7.1 \times 10^{-4}$ \\
\hline
\end{tabular}

Note. Same as for Table 2, except for $f_{s}=3 \times 10^{-5} \mathrm{~Hz}$.

The number of events is expressed in different units. We use $n_{10,10}$ units, which is the number of events $n$ in 10 years-more precisely the number of SMBHBs merging within 10 years for a survey lasting 10 years. The conversion to the number of events per year to merger and per year of survey, $N_{y^{-2}}$, implies a division of $n_{10,10}$ by a factor of 100 . Ad hoc factors have to
Table 4

$\mathrm{S} / \mathrm{Ns}$ for an $N G O$-type Configuration

\begin{tabular}{|c|c|c|c|c|}
\hline$z$ & $\begin{array}{c}M \\
\left(M_{\odot}\right)\end{array}$ & $a$ & $\begin{array}{c}\text { S/N } \\
\text { PN } \\
\text { Inspiral Only }\end{array}$ & $\begin{array}{c}\text { S/N } \\
\text { PhENOMC } \\
\text { Up to Ring Down }\end{array}$ \\
\hline \multirow[t]{2}{*}{0.2} & $2 \times 10^{8}$ & $\begin{array}{r}0 \\
0.9 \\
0.99 \\
\end{array}$ & $\begin{array}{l}1.08 \times 10^{2} \\
1.08 \times 10^{2} \\
1.08 \times 10^{2} \\
\end{array}$ & $\begin{array}{l}1.48 \times 10^{3} \\
3.41 \times 10^{3} \\
4.06 \times 10^{3} \\
\end{array}$ \\
\hline & $2 \times 10^{9}$ & $\begin{array}{r}0 \\
0.9 \\
0.99\end{array}$ & $\begin{array}{l}0 \\
0 \\
0\end{array}$ & $\begin{array}{l}1.08 \times 10^{1} \\
1.84 \times 10^{2} \\
2.86 \times 10^{2}\end{array}$ \\
\hline \multirow[t]{2}{*}{0.3} & $2 \times 10^{8}$ & $\begin{array}{r}0 \\
0.9 \\
0.99 \\
\end{array}$ & $\begin{array}{l}6.17 \times 10^{1} \\
6.17 \times 10^{1} \\
6.17 \times 10^{1} \\
\end{array}$ & $\begin{array}{l}8.73 \times 10^{2} \\
2.02 \times 10^{3} \\
2.41 \times 10^{3} \\
\end{array}$ \\
\hline & $2 \times 10^{9}$ & $\begin{array}{r}0 \\
0.9 \\
0.99 \\
\end{array}$ & $\begin{array}{l}0 \\
0 \\
0\end{array}$ & $\begin{array}{c}0 \\
5.78 \times 10^{1} \\
9.12 \times 10^{1} \\
\end{array}$ \\
\hline \multirow[t]{2}{*}{1} & $2 \times 10^{8}$ & $\begin{array}{r}0 \\
0.9 \\
0.99\end{array}$ & $\begin{array}{l}5.62 \\
5.62 \\
5.62 \\
\end{array}$ & $\begin{array}{l}1.43 \times 10^{2} \\
3.35 \times 10^{2} \\
4.01 \times 10^{2}\end{array}$ \\
\hline & $2 \times 10^{9}$ & $\begin{array}{r}0 \\
0.9 \\
0.99 \\
\end{array}$ & $\begin{array}{l}0 \\
0 \\
0\end{array}$ & $\begin{array}{l}0 \\
0 \\
0\end{array}$ \\
\hline \multirow[t]{2}{*}{1.5} & $2 \times 10^{8}$ & $\begin{array}{r}0 \\
0.9 \\
0.99 \\
\end{array}$ & $\begin{array}{l}0 \\
0 \\
0\end{array}$ & $\begin{array}{l}7.1 \times 10^{1} \\
1.68 \times 10^{2} \\
2.01 \times 10^{2}\end{array}$ \\
\hline & $2 \times 10^{9}$ & $\begin{array}{r}0 \\
0.9 \\
0.99\end{array}$ & $\begin{array}{l}0 \\
0 \\
0\end{array}$ & $\begin{array}{l}0 \\
0 \\
0\end{array}$ \\
\hline
\end{tabular}

Notes. S/Ns (angle-averaged, single mode $l=m=2$ ) relative to $N G O$ for sources located at $z=0.2,0.3,1,1.5$, of mass $2 \times 10^{8}-2 \times 10^{9} M_{\odot}$, and of dimensionless spin Kerr parameter $a=0,0.9,0.99$. The fourth column provides the PN-S/N for the inspiral phase (Poisson \& Will 1995), while the last column provides the phenomenological $\mathrm{S} / \mathrm{N}$ up to the ring-down (PhenomC model; Santamaría et al. 2010), using the conventions by Berti et al. $(2005,2006)$.

be applied, when considering that the SLI lifetime is shorter than the PTA-SKA duration survey (see the Appendix). An example of the third type of unit may be represented by $n_{3,20}$, meaning three years for $\Delta t_{l}$ and twenty years for $\Delta t_{\mathrm{PTA}-\mathrm{SKA}}$, the duration of the PTA-SKA survey. Two simplifying identities appear justified, namely equating (1) $t_{r}$ to $\Delta t_{\mathrm{PTA}-\mathrm{SKA}}$ and (2) the end of the survey by PTA-SKA to the end of the SLI mission (see the Appendix).

\subsection{PTA-SKA Observation Rates (Ideal Sequential Detection)}

Concerning individual observation solely by PTA, Sesana \& Vecchio (2010) estimated that only a handful of observable binaries exist, including those at the $5 \mathrm{~ns}$ effective noise level; the number of resolvable systems quickly drops if the timing precision degrades to, e.g., $50 \mathrm{~ns}$. This result confirms that of Sesana et al. (2009), who analyzed a wide range of population models and indicated 5-15 individual sources having residuals larger than the stochastic background. ${ }^{11}$ The residuals are between 2 and $60 \mathrm{~ns}$ in the frequency range $2 \times 10^{-8}-10^{-7} \mathrm{~Hz}$. Further, they identified most of the individually resolvable SMBHB sources as having a mass larger than $0.5 \times 10^{9} M_{\odot}$ and lying at a redshift $0.2<z<1.5$.

These findings are based on a statistical sample of merging massive galaxies generated from the online Millennium

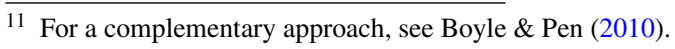


database $^{12}$ built by Springel et al. (2005). The Millennium simulation covers a comoving volume of $\left(500 / h_{100}\right)^{3} \mathrm{Mpc}^{3}$ $\left(h_{100}=H_{0} / 100 \mathrm{~km} \mathrm{~s}^{-1} \mathrm{Mpc}^{-1}\right.$ is the normalized Hubble parameter). Sesana and coworkers populated the merging galaxies with central SMBHs according to different models. For each model, the expected distribution of bright individual sources and the associated timing residuals were computed.

The following procedure has been adopted. Out of the Millennium database, for every cell of the $\left(m_{1}, m_{2}, z\right)$ distribution, we determine the radiating frequency of those SMBHBs which are (1) in an orbital phase at most time $t_{r}$ before merger; (2) radiating at a frequency below $f_{p}$; and (3) generating a timing residual larger than a given threshold. We compute the number of SMBHBs per unit time and per frequency bin (of size equal to $\left.1 / t_{r}\right)$ such that $\mathcal{N}(f)=\int 1 / t_{r} d \mathcal{N}(f) / d f$. Afterward, we integrate $d^{4} \mathcal{N} / d m_{1} d m_{2} d z d t$ along $t_{r}$ and obtain the number of sources for a given bin. Finally, the results are summed up for all bins, masses, and redshifts. The outcome is the number of sources, individually observable by PTA-SKA, merging in time $t_{r}$.

This observation probability depends on the details of the merging SMBHB population, and specifically on the number of coalescing binaries and on their mass. Dependence on other intrinsic binary parameters such as spin and eccentricity may be negligible, though recent studies show that 1.5 post-Newtonian terms, including spin-orbits effects, may be detected (Mingarelli et al. 2012). Indeed, such systems, in the PTA-SKA band, will be far enough from merger for spin-orbit and spin-spin terms not to affect our analysis significantly, but will be close enough to merger, such that the residual eccentricity is likely to be smaller than 0.1 .

Since binaries producing timing residuals in this frequency range are assumed to be extremely massive systems, the rates will depend on the high-mass end of the SMBH mass function. This is an important point since this is precisely where the two most popular massive black hole $(\mathrm{MBH})$ mass predictors give inconsistent results. $\mathrm{MBH}$ masses inferred by the $M-\sigma$ relation (Tremaine et al. 2002; Gultekin et al. 2009; Graham et al. 2011) can indeed be up to an order of magnitude lower with respect to their M-bulge inferred counterparts (Häring \& Rix 2004; Tundo et al. 2007; Lauer et al. 2007; Gultekin et al. 2009).

We have tested nine different models, implementing three different SMBH-host relations and three different accretion prescriptions. On this basis, we have built our catalogue of merging SMBHs starting from the Millennium Run. Nine cases result from the cross-combinations.

The SMBH-host relations explored, including intrinsic scattering (Sesana et al. 2009), are configured in three types.

A. $M-\sigma$ relation according to Tremaine et al. (2002). Following their relation, $\mathrm{SMBH} \propto \sigma^{4}$ implies that SMBHs with masses larger than $10^{9} M_{\odot}$ are extremely rare.

B. $M-\sigma$ relation according to Gultekin et al. (2009). This relation shows a steeper dependence on $\sigma, \mathrm{SMBH} \propto \sigma^{4.24}$; the intrinsic scattering is larger than in Tremaine et al. (2002), predicting more massive binaries.

C. M-bulge relation, again according to Gultekin et al. (2009). The M-bulge relation generally predicts higher $\mathrm{SMBH}$ masses for a given galaxy host; see, e.g., Häring \& Rix (2004), Lauer et al. (2007), and Tundo et al. (2007). In this case, we have $\mathrm{SMBH}$ up to $10^{10} M_{\odot}$.

\footnotetext{
12 Millennium: http://www.g-vo.org/Millennium.
}

Accretion prescriptions (Sesana et al. 2009) are grouped in three classes.

a. Accretion occurs onto the SMBH's remnant, meaning that the two merging SMBHs are undermassive with respect to the selected SMBH-host relation.

b. Accretion occurs onto the primary SMBH before coalescence, meaning that the total mass of the two merging systems follows the SMBH-host relation, but the mass ratio is usually quite high (implying a weaker gravitational wave signal).

c. Accretion occurs onto both SMBHs before coalescence, meaning that the total mass of the two merging systems follows the SMBH-host relation, and the mass ratio of the merging $\mathrm{SMBH}$ is closer to unity (the most favorable situation for gravitational wave detection).

The combination of the above assumptions encompasses a large range of SMBH mass functions, bracketing the observations. The most pessimistic model-Aa-predicts that hardly any SMBHBs with a total mass larger than $10^{9} M_{\odot}$ will coalesce, while the most optimistic model- $\mathrm{Cc}$ - delivers coalescences with a total mass even larger than $10^{10} M_{\odot}$.

Twenty-seven cases are shown; see Table 1 . We consider only SMBHBs that are individually observable by PTA-SKA, generating a timing residual above a given threshold (50, 5, $1 \mathrm{~ns}$ ), and merging in less than 10 years. For these sources, we provide the total number of observations in 10 years in $n_{10,10}$ units (we consider $f_{p}=8 \times 10^{-7} \mathrm{~Hz}$ only). The resolvable SMBHBs are mostly situated at a redshift $0.2<z<1.5$.

The paucity of the rates in Table 1 is far from promising. Further, there is a spread of seven orders of magnitude between the most pessimistic model combined with a $50 \mathrm{~ns}$ timing precision and the most optimistic model combined with a 1 ns timing precision. For a 10 year survey of SMBHBs merging within 10 years, the $\mathrm{Cc}$ scenario predicts $4.7 \times 10^{-2}$ observations at $1 \mathrm{~ns}$, whereas the Aa scenario predicts $3.5 \times 10^{-9}$ at $50 \mathrm{~ns}$. We emphasize that the values in Table 1 include those SMBHBs which would not enter any SLI bandwidth, as they are too massive.

But a different reading of the results is possible, as outlined in the abstract. Indeed, these numbers may be interpreted as ideal upper limits for sequential detection, when assuming (1) an SLI detector with a low cutoff coincident to the high cutoff frequency of PTA-SKA, i.e., $f_{s}=f_{p}$; (2) a noiseless interferometer; under these two conditions, no source would remain undetected by SLI; and finally (3) PTA (or SKA) and SLI operating simultaneously for the same survey duration.

Further, the rates in Table 1 are approximately proportional to the time to merger of the SMBHB, and are obviously proportional to the duration of the survey, so we may switch to $N_{y^{-2}}$ units. Thus, when referring to the conditions (1-3) above, the probability of sequential detection at $1 \mathrm{~ns}$ will never be better than $4.7 \times 10^{-4} \mathrm{yr}^{-2}$, i.e., per year to merger and per year of survey (Cc), or $3.3 \times 10^{-6} \mathrm{yr}^{-2}$ (Aa), since a factor of 100 stands between the two units.

A third presentation of the results may be proposed, when searching for the ideally minimum number of years necessary for a single sequential detection. The number of years will be given by $\sqrt{1 / N_{y^{-2}}}$. At $1 \mathrm{~ns}$, for the Cc scenario we get 46 years versus 550 years for Aa.

This is an extremely optimistic, i.e., fairly unrealistic, scenario for sequential detection, as it implies hypotheses (1) and (2) above, (3) pulsar timing and SLI surveys lasting 46 or even 
550 years, and (4) a precision of $1 \mathrm{~ns}$ (conversely, the number of years may be less, when rates include inspirals with masses lower than $1 \times 10^{8} M_{\odot}$; provided that PTA-SKA is sensitive to lighter masses, the latter have a longer inspiral time). A space mission lasting for decades or centuries is out of the question, but such a constraint does not have not to be fulfilled literally. Indeed, if the mass of the SMBHB can be evidenced from pulsar timing, it is possible to predict when the SMBHB would be visible by an SLI. It would then be the task of the space agencies to launch a mission in time for this rendezvous, a few months or a few centuries after observation by radio astronomy.

It is worth pointing out that the spread among different model predictions decreases as timing precision improves. From Table 1, we conclude that only the precision of $1 \mathrm{~ns}$ timing residuals deserves to be retained for further analysis, as the other precisions are coupled to even more negligible chances.

A caveat is that the population of the merging SMBHBs is constructed out of the Millennium Run. Thus, the total coalescence rate is fixed to that predicted by the Millennium. The actual coalescence rate in the universe is poorly constrained, and it is mostly determined in the high-mass, low-redshift range (relevant to this study), by counting galaxy pairs (Patton et al. 2002; Lin et al. 2004, 2008, 2010; Bell et al. 2006; Xu et al. 2012). The rates predicted by pair counting are consistent with those predicted by semi-analytic merger trees (Volonteri et al. 2003), and by the Millennium Run within a factor of a few (Sesana et al. 2008, 2009). The numbers are, therefore, uncertain by a factor of a few due to different estimates in the merger rate.

Further, the true SMBHB population might not be perfectly described by current models or might come from a completely unexplored physical mechanism (Volonteri 2011).

\subsection{Optimal Sequential Detection Rate}

We now wish to compute how the values in Table 1 are affected by introducing an SLI low cutoff frequency $f_{s}$. The answer depends heavily upon the ring-down frequency of the merging system, Equation (3). Here, we consider three different remnant spins $(a=0,0.9,0.99)$ and two different low cutoff frequencies for SLI $\left(f_{s}=1\right.$ or $\left.3 \times 10^{-5} \mathrm{~Hz}\right)$. We infer the number of possible sequential detections, assuming $1 \mathrm{~ns}$ residual precision. We obtain the values reported in Tables 2 and 3. They are meant as optimal sequential detection rates; the noise characteristics of a given SLI have been ignored. Further, for each column it is assumed that all remnants acquire the same final spin, i.e., the coalescences produce remnants solely of spin $a=0$, or $a=0.9$, or $a=0.99$. Larger rates are associated with higher values of spin.

The optimal sequential detection rates of Tables 2 and 3 are, obviously, lower than the rates of Table 1, because most SMBHs have a ring-down frequency below the $f_{s}$ frequency cutoff. Numbers, in $n_{10,10}$ units, are in the range of $10^{-4}$ to $10^{-2}$, if an SLI low-frequency cutoff at $10 \mu \mathrm{Hz}$ is assumed.

The chances of detection critically drop by about two orders of magnitude when the cutoff is shifted even slightly to higher frequencies, from 10 to $30 \mu \mathrm{Hz}$. Optimal sequential detection rates are in the range of $10^{-6}$ to $10^{-4}$ for an SLI cutoff frequency at $3 \times 10^{-5} \mathrm{~Hz}$. Further, we have tested that the rates are exactly zero in our models for a cutoff at $10^{-4} \mathrm{~Hz}$. The spread in the rate values due to different spins lowers with a decreasing cutoff frequency.

Again, the values may be read differently in $N_{y^{-2}}$ units, obtained by dividing the values in Tables 2 and 3 by a factor of 100 . Finally, a single detection occurs every 79 years for the
Cc scenario, every 587 years for the Aa one, both for a cutoff at $10 \mu \mathrm{Hz}$ and spin of 0.9 , while for a cutoff at $30 \mu \mathrm{Hz}$ and a spin of $0.9,542$ years are required for the $\mathrm{Cc}$ scenario, and 1187 years for the Aa one. The preceding values no longer imply condition (1) in the previous section, but the other conditions hold, i.e., (2) noiseless SLI, (3) pulsar timing and SLI surveys lasting 79 or even 1187 years, or alternatively the possibility of launching an SLI mission at any time within a given period, (4) 1 ns timing precision, and (5) all remnants possessing a spin of 0.9 .

It is legitimate to ask what the rates would be for scenarios displaying multiple spins. Lousto et al. (2010a, 2010b, 2010c) show an analytic distribution for dry mergers following the Kumaraswamy (1980) functional form, peaked at $a=0.75$. For wet mergers, Dotti et al. (2010) provide a statistical distribution, peaked at $a=0.89$. The distribution of cases between dry and wet mergers is not known.

\subsection{Signal-to-Noise Ratio for $N G O$}

We now turn to a specific configuration, namely $N G O$, and attempt to make a more realistic estimate by considering the contribution of noise. Computations relative to other SLI projects will be carried out when such projects will have been planned by space agencies.

For the computation of the $\mathrm{S} / \mathrm{N}$ for $N G O^{13}$ (Jennrich et al. 2011), we stick to the mass range of $2 \times 10^{8}-2 \times 10^{9} \mathrm{M}_{\odot}$, the dimensionless spin Kerr parameter $(a=0,0.9,0.99)$, and introduce four distances $(z=0.2,0.3,1,1.5)$; see Table 4 . Recently, progress has been made on the computation of S/Ns from inspiral to ring-down (Santamaría et al. 2009, 2010; Ajith et al. 2011). Thus, the fifth column in Table 4 shows the $\mathrm{S} / \mathrm{N}$ for phenomenological wave forms, computed with the PHENOMC model (Santamaría et al. 2010). For comparison and for the sole inspiral phase, the fourth column displays the $\mathrm{S} / \mathrm{N}$, computed using a post-Newtonian approximation (Poisson \& Will 1995).

As expected, the computation of $\mathrm{S} / \mathrm{N}$ for $N G O$ shows that part of the sources that have come all the way from the PTA-SKA bandwidth to that of $N G O$ are not necessarily detected by the interferometer, due to the presence of noise. The heaviest SMBHB remnants at $z=0.3$ are not detected if they are associated with a low spin, and for any spin value for $z>0.5$; see Table 4. NGO is optimized for lower mass SMBHBs and this explains the low values of $\mathrm{S} / \mathrm{N}$ for the sources considered herein.

\subsection{Discussion on PTA-SKA and NGO Sequential Detection}

The lowest frequency requirement on NGO (ESA 2011) is $10^{-4} \mathrm{~Hz}$. However, the goal ${ }^{14}$ of $N G O$ is to reach $3 \times$ $10^{-5} \mathrm{~Hz}$ (ESA 2011); further, Jennrich et al. (2011) show that the sensitivity curve stretches below this frequency, down to $10^{-5} \mathrm{~Hz}$. This sensitivity curve has been used for computation of the $\mathrm{S} / \mathrm{N}$; see Table 4 .

To estimate the rates, we must look for a commensurate answer to the spread of several orders of magnitude, due to different astrophysical scenarios (SMBH-host relation, accretion process; for the remnant, fast or slow spin, spin distribution vis-à-vis

\footnotetext{
13 The contribution of confusion noise from white dwarf binaries is marginal.

14 Quoting ESA (2011, p. 74)..."The crucial difference between the requirement and the goal lies in the testing and verification procedures: performances are fully tested and verified against the requirements, whereas goals are observed only in terms of design and analysis, i.e., the mission design must allow for measurements over the wider frequency band. The distinction between goals and requirements is made to prevent excessive efforts on testing and verification, in particular at low frequencies...."
} 
dry and/or wet mergers) and to the paucity of the events. Further, other uncertainties play an important role. Thus, a coarse estimate on the rates is adequate. The other major uncertainties are the following.

In Section 3.2, we showed that a difference of $20 \mu \mathrm{Hz}$ in the SLI low-frequency cutoff determines a difference in the rates (both $n_{10,10}$ and $N_{y^{-2}}$ units) of two orders of magnitude. Moreover, we noted that for a cutoff at $10^{-4} \mathrm{~Hz}$ the chances of sequential detection are null for our simulations. Will $N G O$ perform according to its requirements or its goals? The chances of sequential detection may be meager or simply nonexistent.

The timeline scenario is uncertain at both ends. When will the ambitious 1 ns precision be achieved? Will NGO fly or will there be another SLI-type mission? And when? And of what duration? Let us assume that both conditions, i.e., the residual precision requirement and an SLI launch, will be met in the second half of the next decade. Then, five years may well represent the maximum value for $t_{r}$ that we can observe. When the latter is coupled to, e.g., a three year SLI survey, this implies that the rates in $n_{10,10}$ units must be divided approximatively by a factor $(10 / 5) \times(10 / 3)=6.6$.

Finally, the interferometer noise will further reduce the rates. Altogether, it seems judicious to apply between one and two orders of magnitude of reduction to the rates of Table 2, if $N G O$ goals on sensitivity are met and a cutoff at $10^{-5} \mathrm{~Hz}$ is assumed.

\section{CONCLUSIONS}

We have studied SMBHBs with a mass range of $2 \times 10^{8}-2 \times$ $10^{9} M_{\odot}$. The masses are normalized to a $(1+z)$ factor, the redshift $z$ lying between $z=0.2$ and $z=1.5$, where the individually detectable sources by PTA-SKA are expected to lie. For a high PTA-SKA frequency cutoff of $f_{p}=8 \times 10^{-7} \mathrm{~Hz}$, SMBHBs may pass from the pulsar timing to laser interferometry band in a period ranging from two months to eight years $\left(f_{p}=\right.$ $4 \times 10^{-7} \mathrm{~Hz}$, from 13 months to 50 years). Furthermore, the source signals may be strong enough to be received by both radio astronomical and laser interferometric means.

Unfortunately, the astrophysical estimates act as the showstoppers to sequential detection. Even a noiseless, extremely low-frequency laser space interferometer, coupled to a highly performing pulsar timing of $1 \mathrm{~ns}$, will not allow to go beyond $4.7 \times 10^{-4} \mathrm{yr}^{-2}$ or $3.3 \times 10^{-6} \mathrm{yr}^{-2}$ sequential detections per year to merger and per year of survey, for the most optimistic and pessimistic astrophysical models, respectively. We are dealing with a handful of individually detectable SMBHBs, and therefore it is no surprise that only a very tiny part of those sources merge within a time limit. Other factors reduce the chances even further: higher values of the SLI low cutoff frequency, low precision timing residuals, slowly rotating remnants, and obviously SLI noise. We have also found that the spread between the different astrophysical predictions decreases as timing precision improves, and the SLI low cutoff frequency decreases.

Given the paucity of rates, major changes may come from radically new astrophysical models, or from the achievement of sub-nanosecond precision (predictions may then converge to a rate of $n \times 10^{-1} \mathrm{yr}^{-2}$, per year to merger and per year of survey). For the models, the recent estimates on merger rates and gravitational wave amplitude by McWilliams et al. (2012) are encouraging.

Complementarity between PTA-SKA and SLI should not be dismissed out of hand. Approaching the two bands would be helpful, and efforts to increment the availability of observation time may create favorable conditions to enlarge the PTA bandwidth. SLI should remain interesting for low frequencies in light of the proposed higher frequency interferometers BBO (Phinney et al. 2003) and DECIGO (Seto et al. 2001). An SLI configuration having a cutoff frequency at $10^{-6} \mathrm{~Hz}$ was proposed by Bender (2004).

Opportunities may also lie in inverse search. Ring-down signals observed by SLI may trigger specific searches by PTA-SKA, and hopefully allow the SMBHB to be dug out from the background in the PTA-SKA band. Another opportunity may be provided by observation of PTA-SKA and the consequent evaluation of the SMBHB parameters (mass, spin), leading to a prediction when the merger would occur.

In the absence in the immediate future of a detector covering the gap between PTA-SKA and SLI frequency bands, these efforts might be rewarded. Indeed, the detection of even a single event would be of paramount importance for the understanding of SMBHBs and of the astrophysical processes connected to their formation and evolution.

A. Sesana (Golm) has contributed to the determination of ideal and optimal rates, while E. Berti (Mississipi) to the computation of S/N. Discussions with M. Pitkin (Glasgow), I. Cognard, and K. Liu (Orléans), and exchanges with P. Bender (Boulder), are acknowledged. G. Mamon (Paris) is thanked for his interest in the manuscript.

\section{APPENDIX SCENARIOS}

We define the following times:

1. $t_{b, \mathrm{PTA}-\mathrm{SKA}}$ and $t_{e, \mathrm{PTA}-\mathrm{SKA}}$, the beginning and ending times of the PTA-SKA survey, respectively;

2. $\Delta t_{\mathrm{PTA}-\mathrm{SKA}}$, the duration of the PTA-SKA survey;

3. $t_{0}$, the time of the first observation by PTA-SKA;

4. $t_{p}$, the exit time from the PTA-SKA band;

5. $t_{r}$, the time when the SMBHB's remnant ring-down occurs (approximately the time of merging);

6. $t_{s}$, the entry time in the SLI band;

7. $t_{t}=t_{s}-t_{p}$, the transit time from the PTA-SKA to the SLI band;

8. $t_{l}$, the launch date of the SLI mission;

9. $\Delta t_{l}$, the SLI mission lifetime, which the SLI duration survey, e.g., three years.

With the exception of ideal rates, we have assumed $t_{p} \neq t_{s}$; otherwise, PTA-SKA and SLI would have contiguous bands. Further, $t_{r} t_{s}$ for SMBHB entering the SLI band (otherwise $t_{s}$ is meaningless). Generally, inspiral of SMBHBs is such that $t_{p} \gg t_{0}$. Sequential detection may occur if $t_{l}<t_{r}<t_{l}+\Delta t_{l}$, and for a given $t_{p}$, between two extremes:

1. $t_{r} \rightarrow t_{l+}$ for heavier SMBHBs;

2. $t_{r} \rightarrow\left(t_{l}+\Delta t_{l}\right)_{-}$for lighter SMBHB as they inspiral more slowly.

There are a large variety of operational schedules and potential events associated with PTA-SKA and the SLI mis$\operatorname{sion}(\mathrm{s})$, and to the launch date(s) of the latter. We make no claim here to forecasting events, only to sizing our analysis time wise. We attempt to reasonably group the scenarios into the following types. 

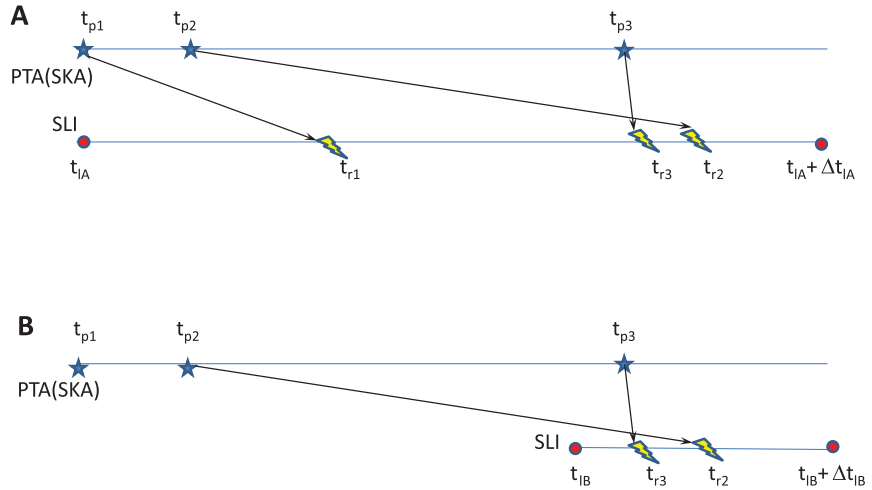

Figure 3. Cases A and B for two different SLI lifetimes. The A case represents an SLI lifetime of the same duration of the PTA-SKA survey, while for the B case, $\Delta t_{l}$ is shorter. The event at $t_{p 1}$ is observed by PTA-SKA but not detected at $t_{r 1}$ by SLI in the B case. For the computation of rates, a reduction factor, given by $t_{l B}-t_{l A}$, is to be applied.

(A color version of this figure is available in the online journal.)

1. $t_{0}$ and $t_{p}$ correspond to a PTA observation occurring in the second half of this decade; $t_{s}$ and $t_{r}$ to an SLI operating in the last quarter of the next decade; this leads to $t_{l}^{\max } \sim 8-15 \mathrm{yr}$ and $\left(t_{l}+\Delta t_{l}\right)^{\max } \sim 11-18 \mathrm{yr}$.

2. $t_{0}$ and $t_{p}$ correspond to a PTA-SKA observation occurring in the first half of the next decade; $t_{s}$ and $t_{r}$ to an SLI operating in the last quarter of the next decade; this leads to $t_{l}^{\max } \sim 3-10 \mathrm{yr}$ and $\left(t_{l}+\Delta t_{l}\right)^{\max } \sim 6-13 \mathrm{yr}$.

3. $t_{0}$ and $t_{p}$, and $t_{s}$ and $t_{r}$ correspond to a PTA-SKA observation and to SLI operations both in the last quarter of the next decade; this leads to $t_{l}^{\max } \sim 0-3 \mathrm{yr}$ and $\left(t_{l}+\Delta t_{l}\right)^{\max } \sim 3-6 \mathrm{yr}$.

4. $t_{0}$ and $t_{p}$ correspond to a PTA-SKA observation occurring in the second half of the next decade; $t_{s}$ and $t_{r}$ to a second-generation SLI operating in the $30 \mathrm{~s}$; this leads to $t_{l}^{\max } \sim 0-15 \mathrm{yr}$ and $\left(t_{l}+\Delta t_{l}\right)^{\max } \sim 3-18 \mathrm{yr}$.

5. Extremely large transit times may also be considered, either for space agencies maintaining in the future a sort of permanent presence of SLI detectors, as is done nowadays in different bands of photon astronomy by successive launches. Alternatively, it may be conceivable to launch an ad hoc SLI mission due to an alert provided by a PTA-SKA observation, a long time before.

We conclude that the largest transit time $t_{t}^{\max }$ for an SMBHB compatible with the scenarios I-IV is in the order of 20 years, while an acceptable average is 10 years. This value is taken as the reference value for our study. The shortest transit time $t_{t}^{\text {min }}$ depends upon the value of $f_{p}$, Equation (1), and it can be safely assumed as a couple of months.

Figure 3 shows how the SLI lifetime affects the rates of sequential detection. The rates in $n_{10,10}$ and $N_{y^{-2}}$ units imply (1) $t_{b, \mathrm{PTA}-\mathrm{SKA}}=t_{l}$ and (2) $t_{e, \mathrm{PTA}-\mathrm{SKA}}=t_{l}+\Delta t_{l}$. If the SLI lifetime is shorter than the duration of the PTA-SKA survey, condition (2) holds and the $n_{10,10}$ rates must be multiplied by the factor $\Delta t_{\mathrm{PTA}-\mathrm{SKA}} \Delta t_{l} / 100$, while the $N_{y^{-2}}$ rates by the factor $\Delta t_{\mathrm{PTA}-\mathrm{SKA}} \Delta t_{l}$.

\section{REFERENCES}

Ajith, P., Hannam, M., Husa, S., et al. 2011, PhRvL, 206, 241101 Amaro-Seoane, P., Aoudia, S., Babak, S., et al. 2012a, arXiv:1201.3621 Amaro-Seoane, P., Aoudia, S., Babak, S., et al. 2012b, CQGra, 29, 124016 Antonucci, F., Armano, M., Audley, H., et al. 2012, CQGra, 29, 124014 Bell, E. F., Phleps, S., Somerville, R. S., et al. 2006, ApJ, 652, 270 Bender, P. L. 2004, CQGra, 21, 1203

Berti, E., Buonanno, A., \& Will, C. M. 2005, PhRvD, 71, 084025
Berti, E., Cardoso, V., \& Will, C. M. 2006, PhRvD, 73, 064030

Bertotti, B. 1984, in ESA-SP-202, SPLAT: Space and Laser Applications and Technology, March 25-30, Les Diablerets (Noordwijk: ESA), 147

Blanchet, L., Spallicci, A., \& Whiting, B. 2011, Mass and Motion in General Relativity, Fundamental Theories of Physics, Vol. 162 (Berlin: Springer) Boyle, L., \& Pen, U.-L. 2010, arXiv:1010.4337

Burt, B. J., Lommen, A. N., \& Finn, L. S. 2011, ApJ, 730, 17

Corbin, V., \& Cornish, N. J. 2010, arXiv:1008.1782

Deng, X., \& Finn, L. S. 2011, MNRAS, 414, 50

Dotti, M., Volonteri, M., Perego, A., et al. 2010, MNRAS, 402, 682

Echeverria, F. 1989, PhRvD, 40, 3194

ESA. 2011, NGO Revealing a Hidden Universe: Opening a New Chapter of Discovery, ESA/SRE(2011)19

Faller, J. E., Bender, P. L., Hall, J. L., Hils, D., \& Stebbins, R. T. 1989, AdSpR, 9, 107

Faller, J. E., Bender, P. L., Hall, J. L., Hils, D., \& Vincent, M. A. 1985, ESA-SP226, Colloquium Kilometric Optical Arrays in Space, October 23-25, 1984 Cargèse (Noordwijk: ESA), 157

Finn, L. S., \& Lommen, A. N. 2010, ApJ, 718, 1400

Forward, R. L., \& Berman, D. 1967, PhRvL, 18, 1071

Graham, A. W., Onken, C. A., Athanassoula, E., \& Combes, F. 2011, MNRAS, 412, 2211

Grassi-Strini, A. M., Strini, G., \& Tagliaferri, G. 1979, NCimL, 24, 212

Gultekin, K., Richstone, D. O., Gebhardt, K., et al. 2009, ApJ, 698, 198

Häring, N., \& Rix, H.-W. 2004, ApJL, 604, L89

Hughes, S. A. 2002, MNRAS, 331, 805

Jenet, F. A., Lommen, A., Larson, S. L., \& Wen, L. 2004, ApJ, 606, 799

Jennrich, O., Petiteau, A., \& Porter, E. 2011, Final Configuration of the ELISA $(N G O)$ Detector for Science Performance Studies, v. 1.2, https://lisa-light.aei.mpg.de/lisa-light/pub/DetectorConfigurations/ FinalConfiguration/ELISA-NGO_FinalConfig.pdf September 21

Kumaraswamy, P. 1980, JHyd, 46, $7 \overline{9}$

Lauer, T. R., Tremaine, S., Richstone, D., \& Faber, S. M. 2007, ApJ, 670, 249

Lee, K. J., Wex, N., Kramer, M., et al. 2011, MNRAS, 414, 3251

Lin, L., Cooper, M. C., Jian, H.-Y., et al. 2010, ApJ, 718, 1158

Lin, L., Koo, D. C., Willmer, C. N. A., et al. 2004, ApJL, 617, L9

Lin, L., Patton, D. R., Koo, D. C., et al. 2008, ApJ, 681, 232

Liu, K., Verbiest, J. P. W., Kramer, M., et al. 2011, MNRAS, 417, 2916

Lommen, A. N., \& Backer, D. C. 2001, ApJ, 562, 297

Lousto, C. O., Campanelli, M., Zochlower, Y., \& Nakano, H. 2010a, CQGra, 27, 114006

Lousto, C. O., Nakano, H., Zochlower, Y., \& Campanelli, M. 2010b, PhRvD, 81,084023

Lousto, C. O., Nakano, H., Zochlower, Y., \& Campanelli, M. 2010c, PhRvD, $82,129902(\mathrm{E})$

McWilliams, S. T., Ostriker, J. P., \& Pretorius, F. 2012, arXiv:1211.4590

Mingarelli, C. M. F., Grover, K., Sidery, T., Smith, R. J. E., \& Vecchio, A. 2012, PhRvL, 109, 081104

Patton, D. R., Pritchet, C. J., Carlberg, R. G., et al. 2002, ApJ, 565, 208

Peters, P. C. 1964, PhRv, 136, B1224

Peters, P. C., \& Mathews, J. 1963, PhRv, 131, 435

Petiteau, A., Babak, S., \& Sesana, A. 2011, ApJ, 732, 82

Phinney, E. S., Bregman, J. N., Carroll, S., et al. 2003, Beyond Einstein: From the Big Bang to Black Holes, NASA Publication NP-2002-10-510-GSFC, http://pcos.gsfc.nasa.gov/docs/Beyond-Einstein.pdf

Pierro, V., \& Pinto, I. 1996, NCimB, 111, 631

Pierro, V., Pinto, I. M., Spallicci, A. D., Laserra, E., \& Recano, F. 2001, MNRAS, 325,358

Pitkin, M. 2012, MNRAS, 425, 2688

Pitkin, M., Clark, J., Hendry, M. A., et al. 2008, J. Phys. Conf. Ser., 122, 012004 Poisson, E., \& Will, C. M. 1995, PhRvD, 52, 848

Rezzolla, L. 2009, CQGra, 26, 094023

Santamaría, L., Krishnan, B., \& Whelan, J. T. 2009, CQGra, 26, 114010

Santamaría, L., Ohme, F., Ajith, P., et al. 2010, PhRvD, 82, 064016

Sesana, A., \& Vecchio, A. 2010, CQGra, 27, 084016

Sesana, A., Vecchio, A., \& Colacino, C. N. 2008, MNRAS, 390, 192

Sesana, A., Vecchio, A., \& Volonteri, M. 2009, MNRAS, 394, 2255

Seto, N., Kawamura, S., \& Nakamura, T. 2001, PhRvL, 87, 221103

Springel, V., White, S. D. M., Jenkins, A., et al. 2005, Natur, 435, 629

Tremaine, S., Gebhardt, K., Bender, R., et al. 2002, ApJ, 574, 740

Tundo, E., Bernardi, M., Hyde, J. B., Sheth, R. K., \& Pizzella, A. 2007, ApJ, 663,57

Volonteri, M. 2011, Journées LISA-France, Laboratoire APC AstroParticules et Cosmologie Paris, May 9-10, http://www.apc.univ-paris7.fr/LISA-France/ 2011-2012_files/Volonteri.pdf

Volonteri, M., Haardt, F., \& Madau, P. 2003, ApJ, 582, 559

Xu, C. K., Zhao, Y., Scoville, N., et al. 2012, ApJ, 747, 85

Yardley, D. R. B., Hobbs, G. B., Jenet, F. A., et al. 2010, MNRAS, 407, 669 